\title{
A precise model for the shape of an adhered microcantilever
}

\author{
Yin Zhang*, Ya-pu Zhao \\ State Key Laboratory of Nonlinear Mechanics, Institute of Mechanics, Chinese Academy of Sciences, Beijing 100190, China
}

\section{A R T I C L E I N F O}

\section{Article history:}

Received 20 November 2010

Received in revised form 15 May 2011

Accepted 1 September 2011

Available online 12 September 2011

\section{Keywords:}

Stiction

Microcantilever

Adhesion

Arc-shape

S-shape

\begin{abstract}
A B S T R A C T
A variational method using the principle of virtual work (PVW) is presented to formulate the problem of the microcantilever stiction. Compared with the Rayleigh-Ritz method using the arc-shaped or S-shaped deflection, which prescribes the boundary conditions and thus the deflection shape of a stuck cantilever beam, the new method uses the matching conditions and constraint condition derived from PVW and minimization of the system free energy to describe the boundary conditions at the contact separation point. The transition of the beam deflection from an arc-shape-like one to an S-shape-like one with the increase of the beam length is shown by the new model. The (real) beam deflection given by this new model deviates more or less from either an arc-shape or an S-shape, which has significant impact on the interpretation of experimental data. The arc-shaped or S-shaped deflection assumption ignores the beam bending energy inside the contact area and the elastic energy due to the beam/substrate contact, which is inappropriate as shown by this study. Furthermore, the arc-shaped or S-shaped deflection only approximately describes the deflection shape of a stuck beam with zero external load and obviously, the external load changes the beam deflection. The Rayleigh-Ritz method using the arc-shaped or S-shaped deflection assumption in essence can only be used to tell approximately whether stiction occurs or not. Rather than assuming a certain deflection shape and by incorporating the external load, the new method offers a more general and accurate study not only on the microcantilever beam stiction but also on its de-adherence.
\end{abstract}

(C) 2011 Elsevier B.V. All rights reserved.

\section{Introduction}

Stiction is one of the most widespread hazards threatening the reliable operation of the microelectromechanical systems (MEMS) devices [1,2]. Stiction is often categorized as release-related stiction and in-use stiction [1,3]. The capillary force [1,2,4,6-9], electrostatic force [4,9-13], mechanical load [14] and inertial forces [8] during the MEMS release or in-use stage can all be the actuation mechanisms to bring the devices into contact with one another or with the substrate. The system free energy of the device in contact consists of two parts: the mechanical energy and surface energy. Adhesion energy is defined as the reduction of the surface energy per unit area when combining two surfaces into one interface [15], which is also referred to as the surface interaction energy [1,2]. Once the external load is retracted, two things happen in terms of the system free energy: the increase of the mechanical energy due to the device deformation and the decrease of the surface energy due to adhesion. In terms of force, the device deformation generates a restoring force to try to pull the device back to the free-standing state; the tensile pressure around the contact separation edge $[16,17]$ due to

\footnotetext{
* Corresponding author. Tel.: +86 10 82543970; fax: +86 1082543935 .

E-mail address: zhangyin@lnm.imech.ac.cn (Y. Zhang).
}

adhesion tries to balance the restoring force and keeps the devices in a deformed state. The competition between the mechanical and surface energies determines whether stiction occurs or not. Here the stiction state is defined as an attachment state after the external/actuation load is retracted. Therefore, the external load does not appear as a parameter in many stiction studies $[7,8,18]$. The stable equilibrium of a stiction state corresponds to a local minimum of the system total free energy $[5,18]$ and there is no stiction if such local minimum does not exist [18]. The peel number of $N_{P}$ [18], which is defined as a convenient way to tell whether stiction occurs or not, is given as follows for a cantilever beam

$N_{P}=C \frac{E_{1} T^{3} H^{2}}{\gamma_{s} L_{u}^{4}}$

$C$ is a constant. $E_{1}, T$ and $L_{u}$ are Young's modulus, thickness and unstuck length of the cantilever, respectively. $H$ is the gap distance between the undeformed beam and substrate as shown in Fig. 1(a). $\gamma_{s}$ is the adhesion energy. Stiction occurs when $N_{P} \leq 1$ and no stiction when $N_{P}>1$ [18]. $N_{P}=1$ corresponds to the cantilever equilibrium obtained by minimizing the system free energy as shown in Appendix A. The dimensionless peel number can also be viewed as the order of the ratio of the mechanical energy to the surface energy $[2,19]$. By setting $N_{P}=1$, a critical length, $L_{\text {crit }}$, which 
(a) Schematic diagram of a stuck cantilever beam

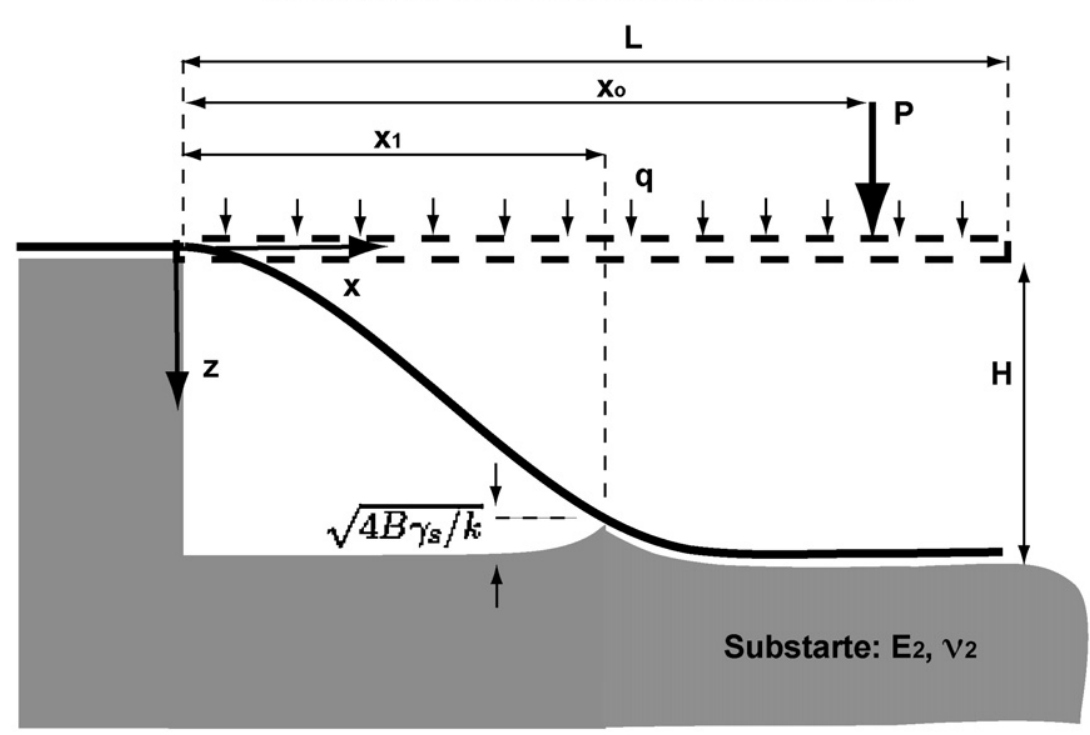

(b) Arc and S-shaped deformations

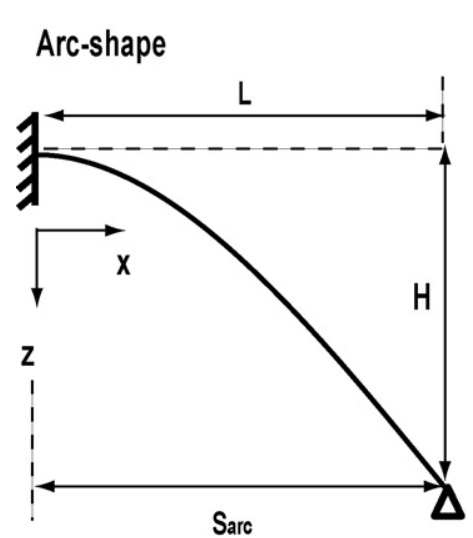

Sarc $=\mathrm{L}$
S-shape

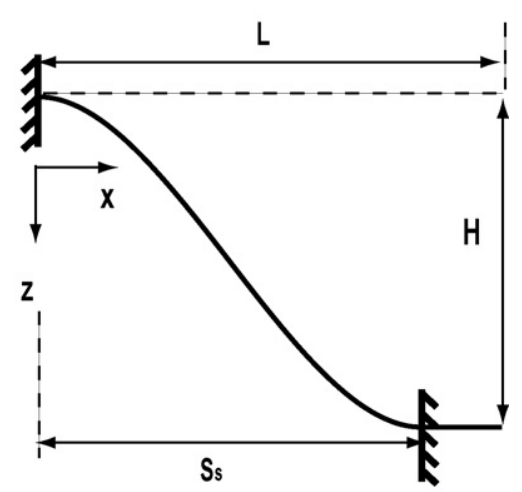

$\mathrm{S}_{\mathrm{s}}<\mathrm{L}$

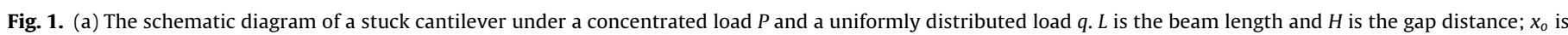
the location of $P$ and $x_{1}$ is the separation point. (b) The arc-shaped and S-shaped deflections. $S_{\text {arc }}$ and $S_{S}$ are the unstuck lengths of an arc-shape and an S-shape.

is variably referred to as the detachment length $[4,7,18]$ or as the length of the shortest adhered beam [20], is obtained as follows:

$L_{\text {crit }}=\sqrt[4]{C \frac{E_{1} T^{3} H^{2}}{\gamma_{s}}}$

When $E_{1}, T, H$ and $\gamma_{s}$ are fixed, the cantilever beam with a length smaller than this critical length peels-off from the substrate; otherwise, the beam is stuck. Eq. (2) can also be rewritten as follows to quantify the adhesion energy of $\gamma_{s}$ in those experiments using the cantilever array with different lengths $[8-10,18,20]$

$\gamma_{s}=C \frac{E_{1} H^{2} T^{3}}{L_{c r i t}^{4}}$

Eqs. (1)-(3) are three different but equivalent ways of characterizing the beam stiction. The key issue in these three equations is the calculation of the system mechanical energy, which is embodied in the constant of $C$. $C=3 / 8$ if the cantilever is assumed to have an arc-shaped deflection [3,4,18]; $C=3 / 2$ if the cantilever is assumed to have an $S$-shaped deflection $[5,8,9]$. Obviously, different $C$ s have significant impact on the cantilever stiction criteria as indicated by the above three equations. For example, four times difference of $\gamma_{s}$ results from the different $C$ s of an arc-shape and an S-shape as indicated by Eq. (3). The arc-shaped and S-shaped deflections are shown in Fig. 1(b) and their derivation is given in Appendix A. The arc-shaped and S-shaped deflection shapes (assumptions) have been the cornerstone in the above beam stiction studies. The description on the formation of arc- and S-shape is no more than the following (vague) summary [20]: longer beam forms an S-shape and its unstuck length is appreciably shorter than the beam length; shorter beam forms an arc-shape and its unstuck length is approximately equal to the beam length. However, none of the above three equations can tell when an arc-shape or an S-shape is formed for a beam with a given length. On the contrary, the above three equations actually indicate that the beam length $(L)$ has nothing to do with the peel number, detachment length and adhesion energy measurement, respectively. How the beam dimensions together with the adhesion energy and external load influence the stuck beam deflection shape remains as an unanswered question in the previous studies.

Legtenberg et al. [7] applied the Rayleigh-Ritz method to calculate the mechanical energy of a stuck clamped-clamped beam 
by assuming its deflection shape. The Rayleigh-Ritz method [21] is an approximate method whose accuracy depends on the assumed deflection shape. The arc-shape and S-shape as indicated by their names also assume the cantilever deflection shape by prescribing certain boundary conditions at the contact separation point as shown in Appendix A. The method with the arc-shape or S-shape assumption in essence is the Rayleigh-Ritz method. The boundary conditions at the contact separation point are (assumed) hinged for an arc-shape and (assumed) clamped for an S-shape. There are some arguments on the choice of arc-shape and S-shape in the cantilever stiction study. Because $C=3 / 8$ for an arc-shape and $C=3 / 2$ for an $S$-shape, $L_{\text {crit }}$ of an arc-shape is a shorter one. $L_{c r i t}$ is found from the stuck beam with the shortest length in the cantilever beam array experiments $[10,20]$. Therefore, an arc-shape is a natural choice in those cantilever beam array experiments $[3,4,18]$. However, it is found that the experimentally measured adhesion energies from the arc-shaped and S-shaped cantilevers are consistently different $[1,8]$. de Boer and Michalske [8] argued that (only) "the S-shaped cantilever beam configuration should be adopted for detailed studies of adhesion forces in surface micromachining". Furthermore, Yang [5] argued that the boundary conditions at the contact separation point cannot be the hinged ones, which is another way of saying that the arc-shaped deflection cannot be formed. The arc-shaped deflection is an idealized point contact scenario as analyzed in Appendix A, whose small contact area can introduce significant statistical errors to impact the experimental results and interpretation [1]. An arc-shape and an S-shape are the stuck cantilever deflections under zero external load as shown in Appendix A. Once an actuation force such as an electrostatic force [4,9-13] or a mechanical load [14] is exerted, the beam deflection will deviate more or less from an arc-shape or an S-shape, which directly impacts the calculation of the system mechanical energy. In the de-adherence studies of the stuck beams [11-13], the beam deflection under different loads needs to be closely monitored to tell whether the de-adherence occurs or not. Fixing the beam deflection shape as an arc-shape or an S-shape is obviously not a good choice, which can cause significant error. Yang incorporated a uniformly distributed load in the stiction study of a clamped-clamped beam [5]. However, Yang's energy approach, which also prescribes the boundary conditions at the separation points, shows that there is no difference between a compressive load (pushing down) and a tensile load (pulling up) as reflected in Yang's Eqs. (16) and (29)[5].

The energy approach $[5,8,18]$ and the fracture mechanics approach [8-10,14], which either assumes an arc/S-shaped deflection or specifies the boundary conditions, are two major methods in the beam stiction study. In the energy approach, the total system free energy $U_{T}=U_{B}+U_{S}$ ( $U_{B}$ is the mechanical energy of a bending beam and $U_{S}$ is its surface energy) and the equilibrium is obtained by requiring $\partial U_{T} / \partial a=0$ ( $a$ is the (unknown) contact length) $[5,18]$. In the fracture approach, $\partial U_{B} / \partial a$ yields a quantity called the energy release rate and $\partial U_{B} / \partial a=\gamma_{s}$ is required when there is no crack propagation [8-10,14]. $\partial U_{T} / \partial a=0$ and $\partial U_{B} / \partial a=\gamma_{s}$ yield the same results as shown by de Boer and Michalske [8]. Both the energy method $[5,8,18]$ and the fracture mechanics method $[8-10,14]$ only account for the mechanical energy of the bending beam in the unstuck part; the mechanical energy inside the contact area including the bending energy and elastic energy due to the beam/substrate contact deformation are not included in these two methods. It is reasonable to ignore the beam bending energy inside the contact area if an arc-shaped or S-shaped deflection is assumed: an arc-shape is a point contact and the deflection of an S-shape is a flat one inside the contact area. However, we show that a point contact cannot occur in adhesive contact and the (long) beam deflection inside the contact area is not exactly flat. The energy method [5] and the fracture mechanics method [8] are more or less inspired by Obreimoff's classical paper in 1930 on the mica splitting test [22], which does not account for the mechanical energy inside the contact area, either. There is one critical difference in the adhesion energy between the Obreimoff's mica splitting test and the microcantilever beam stiction test. Obreimoff's splitting test of mica which is atomically smooth is actually the cleavage of mica and the adhesion energy is "the extremely high value" of $2 \times 10^{4} \mathrm{~mJ} / \mathrm{m}^{2}$ [22]. This large adhesion energy makes the deformation of the mica lamina inside contact area (unsplit part) extremely small compared with that of the split part. However, the real contact area in MEMS stiction is a small portion of the nominal area due to the surface roughness $[1,2]$. The (nominal) adhesion energy measured by the (hydrophilic) polysilicon beam stiction test ranges from $0.25 \mathrm{~mJ} / \mathrm{m}^{2}$ [20] to $270 \pm 100 \mathrm{~mJ} / \mathrm{m}^{2}$ [18], which is several orders of magnitude smaller than that of mica. At the same time Young's modulus of polysilicon (170 GPa [18]) is similar to that of mica (196 GPa [22]). It is a safe way to incorporate the mechanical energy inside the contact area into the system free energy in the study of MEMS stiction.

The inconsistency and unreliability of the experimental data obtained in the beam stiction test have been noticed $[1,23]$. van Spengen et al. [1] concluded that "the surface interaction energy measurement using stuck beams needs considerably more research before we can conclude anything definite about the precise magnitude of the measured surface interaction energy". Most of the previous studies, according to van Spengen et al. [2], have "never come further than a peel number". A more comprehensive way of studying the beam stiction should include the effect of surface roughness $[1,2,23]$, whose distribution determines how two surfaces contact each other. A more accurate description on the stuck beam deflection in essence only offers a better characterization of the nominal adhesion energy. However, it is still a valuable tool and allows us to observe trends [1]. The principle of virtual work (PVW) is used in this study to derive the governing equation and matching/boundary conditions of a stuck cantilever. Unlike that an arc-shape or an S-shape specifies the boundary conditions at the contact separation points, the matching conditions determine what kind of the boundary conditions should be formed at the contact separation point, which are neither hinged nor clamped. The model presented here incorporates the cantilever beam dimensions, adhesion and external load and shows how these quantities change the beam deflection shape rather than prescribing it. By doing so, a more general and accurate method of describing the stuck cantilever deflection is presented.

\section{Model development}

Fig. 1(a) shows a cantilever beam under a concentrated load $P$ and a uniformly distributed load $q$. The coordinate system is also shown in the figure. The governing equations are different for the free-standing and stiction states. For brevity, the governing equation for the free-standing state is directly given as follows [24,25]:

$E_{1} I \frac{d^{4} W}{d x^{4}}=P \delta_{D}\left(x-x_{0}\right)+q$

$E_{1}$ is the beam Young's modulus, $I$ is the area moment of inertia defined as $I=B T^{3} / 6$ ( $2 B$ is the beam width and $T$ is the beam thickness). $W=W(x)$ is the beam deflection. $\delta_{D}\left(x-x_{0}\right)$ is the Dirac delta function and $x_{o}$ is the location of the concentrated force $P$. The following boundary conditions hold for a cantilever beam:

$W(0)=0, \quad \frac{d W}{d x}(0)=0, \quad \frac{d^{2} W}{d x^{2}}(L)=0, \quad \frac{d^{3} W}{d x^{3}}(L)=0$

$L$ is the beam length. The above four equations indicate the zero displacement and slope at the fixed end and zero moment and shear at the free end. $W$ is solved by integrating Eq. (4) and applying the 


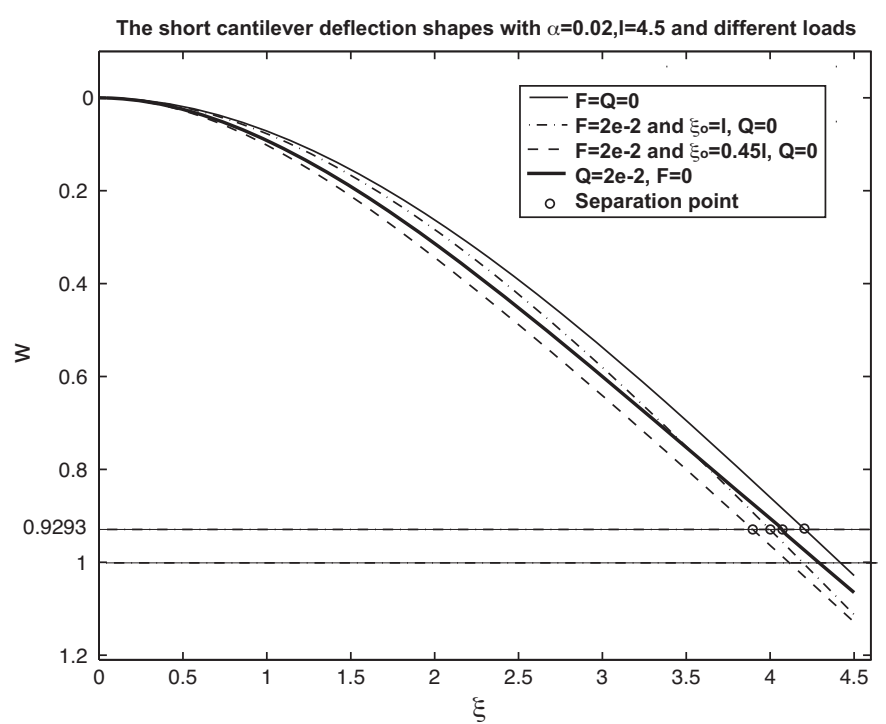

Fig. 2. The deflection shapes of a "chunky" beam with $l=4.5$ and $\alpha=0.02$ under four different loading scenarios: (1) $F=Q=0$ (stiction state). (2) $F=2 \times 10^{-2}$ and $\xi_{0}=l, Q=0$ (concentrated force inside contact zone). (3) $F=2 \times 10^{-2}$ and $\xi_{o}=0.45 l, Q=0$ (concentrated force outside contact zone). (4) $Q=2 \times 10^{-2}$ and $F=0$ (uniformly distributed load).

boundary conditions of Eq. (5) as follows:

$W= \begin{cases}\frac{P}{6 E_{1} I}\left(3 x_{0} x^{2}-x^{3}\right)+\frac{q}{E_{1} I}\left(\frac{L^{2} x^{2}}{4}-\frac{L x^{3}}{6}+\frac{x^{4}}{24}\right), & 0 \leq x \leq x_{o} \\ \frac{P}{6 E_{1} I}\left(3 x_{o}^{2} x-x_{0}^{3}\right)+\frac{q}{E_{1} I}\left(\frac{L^{2} x^{2}}{4}-\frac{L x^{3}}{6}+\frac{x^{4}}{24}\right), & x_{o}<x \leq L\end{cases}$

When the beam is stuck with the substrate, the beam deflection is divided into two parts as follows:

$W= \begin{cases}W_{1}, & 0 \leq x \leq x_{1} \\ W_{2}, & x_{1} \leq x \leq L\end{cases}$

As shown in Fig. 1(a) $x_{1}$ is the point where the beam separates from the substrate, which is also the crack tip locations from the viewpoint of fracture mechanics [8-10,14]. $W_{1}$ is the beam displacement in the suspension/unstuck zone and $W_{2}$ is the beam displacement in the contact/stuck zone. When a beam under a concentrated load, there is a contact scenario called discontinuous contact $[24,26]$ in which there are multiple contact zones. In non-adhesive contact, the concentrated compressive load required for the discontinuous contact is so large that the beam breaks down long before this load can be reached [24]. However, in adhesive contact, a beam can take a tensile load and the cantilever free end may separate from the substrate, which makes the above assumption of two zones invalid. The limitation of Eq. (7) will be discussed later in details.

The bending energy $U_{B}$, which consist of both unstuck and stuck parts, is the following

$U_{B}=\frac{E_{1} I}{2} \int_{0}^{L} \frac{d^{2} W}{d x^{2}} d x=\frac{E_{1} I}{2}\left(\int_{0}^{x_{1}} \frac{d^{2} W_{1}}{d x^{2}} d x+\int_{x_{1}}^{L} \frac{d^{2} W_{2}}{d x^{2}} d x\right)$

$U_{F}$ of the energy stored by the elastic foundation due to the beam/substrate contact is given as follows:

$U_{F}=\frac{k}{2} \int_{x_{1}}^{L}\left(W_{2}-H\right)^{2} d x$

$H$ is the gap distance between the undeformed beam and substrate as shown in Fig. 2(a) and $k$ is the modulus of elastic foundation. Eq. (9) indicates that the potential energy is stored by a series of springs with stiffness $k$. For a rectangular beam indenting a substrate which is modeled as an elastic half space, $k$ is given as follows [27]

$k=0.71 E_{2}\left(\frac{E_{2} B^{4}}{E_{1} I}\right)^{1 / 3}=1.28 E_{2}\left(\frac{B}{T}\right)\left(\frac{E_{2}}{E_{1}}\right)^{1 / 3}$

$E_{2}$ is Young's modulus of substrate.

The surface energy, $U_{S}$, is given as the following $[5,7,18]$

$U_{S}=-2 B \gamma_{S}\left(L-x_{1}\right)$

$2 B$ is the beam width and $L-x_{1}$ is the beam contact length $2 B\left(L-x_{1}\right)$ is thus the contact/stuck area. $\gamma_{s}$ is the adhesion energy between the beam and substrate, which is also known as the surface interaction energy [1,2] and the Dupré work of adhesion [19]. It is noticed that $U_{S}$ is negative, which physically means that the system free energy reduces when combining two surfaces into one interface [15]. This reduction of surface energy is the mechanism responsible for the microstructure stiction. On the other side, the restoring force due to the mechanical energy $\left(U_{B}\right.$ and $\left.U_{F}\right)$ tries to pull the beam back to the free-standing state. $U_{\text {force }}$, the work done by $P$ and $q$, is given as follows:

$U_{\text {force }}=\int_{0}^{W} q d W+\int_{0}^{W} P \delta_{D}\left(x-x_{0}\right) d W$

By applying the principle of virtual work (PVW) [25,26], i.e., $\delta\left(U_{B}+U_{F}+U_{S}-U_{\text {force }}\right)=0$, the following matching/boundary conditions at $x=x_{1}$ are obtained

$$
\begin{aligned}
& W_{1}\left(x_{1}\right)=W_{2}\left(x_{1}\right), \quad \frac{d W_{1}}{d x}\left(x_{1}\right)=\frac{d W_{2}}{d x}\left(x_{1}\right), \quad \frac{d^{2} W_{1}}{d x^{2}}\left(x_{1}\right)=\frac{d^{2} W_{2}}{d x^{2}}\left(x_{1}\right), \\
& \frac{d^{3} W_{1}}{d x^{3}}\left(x_{1}\right)=\frac{d^{3} W_{2}}{d x^{3}}\left(x_{1}\right)
\end{aligned}
$$

It is noticed that the same matching conditions are also obtained by Ghatak et al. [28]. The boundary conditions at $x=0$ and $x=L$ are also obtained by PVW as follows:

$W_{1}(0)=0, \quad \frac{d W_{1}}{d x}(0)=0, \quad \frac{d^{2} W_{2}}{d x^{2}}(L)=0, \quad \frac{d^{3} W_{2}}{d x^{3}}(L)=0$

It is noticed that the adhesion energy $\left(\gamma_{s}\right)$ and the elastic foundation modulus $(k)$ have no impact on the cantilever boundary conditions, which are the same as Eq. (5). The governing equation is also derived from PVW. Because the concentrated force $P$ can be either inside the contact zone or outside the contact zone, there are two sets of governing equations for these two scenarios. When $P$ is inside the contact zone, i.e., $x_{0}>x_{1}$, the governing equation is as follows:

$$
\begin{cases}E_{1} I \frac{d^{4} W_{1}}{d x^{4}}=q, & 0 \leq x \leq x_{1} \\ E_{1} I \frac{d^{4} W_{2}}{d x^{4}}+k\left(W_{2}-H\right)=q+P \delta_{D}\left(x-x_{0}\right), & x_{1} \leq x \leq L\end{cases}
$$

The previous receding contact mechanics models explicitly or implicitly assume that the concentrated load is inside the contact area [24-26,29]. However, the concentrated load can be outside the contact area. For example, the concentrated force exerted by a nanoindenter in Jones' experiment [14] is outside the contact area. When $P$ is outside the contact zone, i.e., $x_{0}<x_{1}$, the governing equation is as follows:

$\begin{cases}E_{1} I \frac{d^{4} W_{1}}{d x^{4}}=q+P \delta_{D}\left(x-x_{0}\right), & 0 \leq x \leq x_{1} \\ E_{1} I \frac{d^{4} W_{2}}{d x^{4}}+k\left(W_{2}-H\right)=q, & x_{1} \leq x \leq L\end{cases}$

Keep in mind that the separation point of $x_{1}$ is unknown. The total free energy of the system is

$U_{T}=U_{B}+U_{F}+U_{S}$ 
For equilibrium, $d U_{T} / d x_{1}$ vanishes giving $[5,7,16,18]$

$-\frac{k}{2}\left[W_{2}\left(x_{1}\right)-H\right]^{2}+2 B \gamma_{s}=0$

To derive Eq. (18), the derivative properties such as $\frac{d}{d x_{1}}\left(\int_{0}^{x_{1}} \frac{d^{2} W_{1}}{d x^{2}} d x\right)=\frac{d^{2} W_{1}}{d x^{2}}\left(x_{1}\right), \quad \frac{d}{d x_{1}}\left(\int_{x_{1}}^{L} \frac{d^{2} W_{2}}{d x^{2}} d x\right)=-\frac{d^{2} W_{2}}{d x^{2}}\left(x_{1}\right)$ and $\frac{d}{d x_{1}}\left[\int_{x_{1}}^{L}\left(W_{2}-H\right)^{2} d x\right]=\left[W_{2}\left(x_{1}\right)-H\right]^{2} \quad$ together with $\frac{d^{2} W_{1}}{d x^{2}}\left(x_{1}\right)=\frac{d^{2} W_{2}}{d x^{2}}\left(x_{1}\right)$ of matching condition are applied. $W_{2}\left(x_{1}\right)$ is readily solved from Eq. (18) as $W_{2}\left(x_{1}\right)=H \pm \sqrt{4 B \gamma_{s} / k}$. Because the elastic foundation does not allow the sink-in phenomenon, which corresponds to the solution of $W_{2}\left(x_{1}\right)=H+\sqrt{\frac{4 B \gamma_{s}}{k}}$ [29], $W_{2}\left(x_{1}\right)$ can only have the following solution

$W_{2}\left(x_{1}\right)=H-\sqrt{\frac{4 B \gamma_{s}}{k}}=H-1.77 \sqrt{\frac{T \gamma_{s}}{E_{2}}\left(\frac{E_{1}}{E_{2}}\right)^{1 / 3}}$

For non-adhesive contact of $\gamma_{s}=0$, Eq. (19) recovers the constraint condition of reference [25]. From the viewpoint of fracture mechanics, this $\sqrt{4 B \gamma_{s} / k}\left(\right.$ or $1.77 \sqrt{T \gamma_{s} / E_{2} \times\left(E_{1} / E_{2}\right)^{1 / 3}}$ ) as shown in Fig. 1(a) is the critical normal crack opening displacements [29].

The following nondimensionalization scheme is introduced [24-26]

$$
\begin{gathered}
\xi=\beta x, \quad w_{i}=\beta W_{i}(i=1,2), \quad \xi_{1}=\beta x_{1}, \quad h=\beta H, \quad l=\beta L, \\
F=\frac{P}{4 \beta^{2} E_{1} I}, \quad Q=\frac{q}{\beta^{3} E_{1} I}
\end{gathered}
$$

$\beta$ is defined as $[24,25]$

$\beta=\sqrt[4]{\frac{k}{4 E_{1} I}}$

$\beta$ has the unit of $\mathrm{m}^{-1}$. Substitute $k$ of Eq. (10) into Eq. (21), $\beta$ is found as

$\beta \approx 1.18\left(\frac{E_{2}}{E_{1}}\right)^{1 / 3} \frac{1}{T}$

For two solids with similar Young's moduli (i.e., $E_{1} \approx E_{2}$ ), $\beta \approx 1 / T$, which is to say that the nondimensionalization scheme of Eq. (20) on the beam dimensions is to give the ratios of the beam dimensions to its thickness. For example, $l=\beta L \propto L / T$ indicates the slenderness of beam. Now Eq. (6), the beam solution of the free-standing state under a concentrated load and uniformly distributed load, is nondimensionalized as the following

$w= \begin{cases}\frac{2 F}{3}\left(3 \xi_{0} \xi^{2}-\xi^{3}\right)+Q\left(\frac{l^{2} \xi^{2}}{4}-\frac{l \xi^{3}}{6}+\frac{\xi^{4}}{24}\right), & 0 \leq x \leq x_{0} \\ \frac{2 F}{3}\left(3 \xi_{0}^{2} \xi-\xi_{0}^{3}\right)+Q\left(\frac{l^{2} \xi^{2}}{4}-\frac{l^{3}}{6}+\frac{\xi^{4}}{24}\right), & x_{0}<x \leq L\end{cases}$

The matching conditions of Eq. (13) are nondimensionalized as follows:

$$
\begin{gathered}
w_{1}\left(\xi_{1}\right)=w_{2}\left(\xi_{1}\right), \quad \frac{d w_{1}}{d \xi}\left(\xi_{1}\right)=\frac{d w_{2}}{d \xi}\left(\xi_{1}\right), \quad \frac{d^{2} W_{1}}{d \xi^{2}}\left(\xi_{1}\right)=\frac{d^{2} W_{2}}{d \xi^{2}}\left(\xi_{1}\right), \\
\frac{d^{3} w_{1}}{d \xi^{3}}\left(\xi_{1}\right)=\frac{d^{3} w_{2}}{d \xi^{3}}\left(\xi_{1}\right)
\end{gathered}
$$

The boundary conditions of Eq. (14) are now nondimensionalized as

$w_{1}(0)=0, \quad \frac{d w_{1}}{d \xi}(0)=0, \quad \frac{d^{2} W_{2}}{d \xi^{2}}(l)=0, \quad \frac{d^{3} w_{2}}{d \xi^{3}}(l)=0$
Eq. (19), the constraint condition of adhesive contact, is nondimensionalized as follows:

$w_{2}\left(\xi_{1}\right)=h-\frac{\sqrt{\alpha}}{2}$

$\alpha$ is a dimensionless parameter defined as

$\alpha=\frac{4 B \gamma_{s}}{E_{1} I \beta^{2}}=\frac{24 \gamma_{s}}{E_{1} T^{3} \beta^{2}}$

Eq. (15), the governing equation of the concentrated load inside the contact zone, is nondimensionalized as follows:

$\begin{cases}\frac{d^{4} w_{1}}{d \xi^{4}}=Q, & 0 \leq \xi \leq \xi_{1} \\ \frac{1}{4} \frac{d^{4} w_{2}}{d \xi^{4}}+w_{2}-h=\frac{Q}{4}+F \delta_{D}\left(\xi-\xi_{0}\right), & \xi_{1} \leq \xi \leq l\end{cases}$

Eq. (16), the governing equation of the concentrated load outside the contact zone, is nondimensionalized as

$$
\begin{cases}\frac{d^{4} w_{1}}{d \xi^{4}}=Q+4 F \delta_{D}\left(\xi-\xi_{0}\right), & 0 \leq \xi \leq \xi_{1} \\ \frac{1}{4} \frac{d^{4} w_{2}}{d \xi^{4}}+w_{2}-h=\frac{Q}{4}, & \xi_{1} \leq \xi \leq l\end{cases}
$$

The solution to Eq. (28) as given in the following equation consists of two parts: homogeneous solution and particular solution. The difficulty is to find the particular solution and Weitsman gave the detailed procedures of deriving the particular solution [24]

$$
w= \begin{cases}w_{1}=A_{1} \xi^{3}+B_{1} \xi^{2}+C_{1} \xi+D_{1}+\frac{Q}{24} \xi^{4}, & 0 \leq \xi \leq \xi_{1} \\ w_{2}=A_{2} \cosh \xi \sin \xi+B_{2} \cosh \xi \cos \xi+C_{2} \sinh \xi \sin \xi & \\ +D_{2} \sinh \xi \cos \xi-\frac{F}{2} \sinh \left|\xi-\xi_{0}\right| \cos \left(\xi-\xi_{0}\right) & \\ F & \\ +\frac{F}{2} \cosh \left(\xi-\xi_{0}\right) \sin \left|\xi-\xi_{0}\right|+\frac{1}{4} Q+h, & \xi_{1} \leq \xi \leq l\end{cases}
$$

It is worth pointing out that Eq. (30) is different from the solution given by Weitsman [24]. The beam studied by Weitsman is an infinite one and the vanishing of bending moment is assumed at the contact separation, which in general is inappropriate and a general solution is given by Zhang and Murphy [25,29]. As mentioned above, Yang argued that the bending moment at the contact separation point cannot be zero in an adhesive contact [5]. The solution of Eq. (30) does not prescribe any specific conditions at the contact separation point of $\xi_{1}$. Eq. (30) is also different from those solutions given in Refs. $[25,29]$ because of the different contact scenarios and coordinate systems. However, the ideas of deriving the beam solution with a concentrated inside the contact zones in those Refs. $[24,25,29]$ apply.

The solution to Eq. (29) of the concentrated force outside the contact zone is now derived as follows:

$w= \begin{cases}w_{1}=A_{1} \xi^{3}+B_{1} \xi^{2}+C_{1} \xi+D_{1}+\frac{Q}{24} \xi^{4}+4 F H_{3}\left(\xi-\xi_{0}\right), & 0 \leq \xi \leq \xi_{1} \\ w_{2}=A_{2} \cosh \xi \sin \xi+B_{2} \cosh \xi \cos \xi+C_{2} \sinh \xi \sin \xi & \\ +D_{2} \sinh \xi \cos \xi+\frac{1}{4} Q+h, & \xi_{1} \leq \xi \leq l\end{cases}$

$H_{3}\left(\xi-\xi_{0}\right)$ is a function defined as follows:

$H_{3}\left(\xi-\xi_{0}\right)=\int_{0}^{l} \int_{0}^{l} \int_{0}^{l} \int_{0}^{l} \delta_{D}\left(\xi-\xi_{0}\right) d \xi d \xi d \xi d \xi= \begin{cases}0, & \xi-\xi_{0} \leq 0 \\ \frac{\left(\xi-\xi_{0}\right)^{3}}{6}, & \xi-\xi_{0}>0\end{cases}$

$A_{i}, B_{i}, C_{i}$ and $D_{i}(i=1$ and 2$)$ are the eight unknown constants to be determined. These eight unknowns together with $\xi_{1}$ are the nine unknowns in total to be solved. Eqs. (24)-(26) offer nine equations in total to solve these nine unknowns. Because $\xi_{1}$, the separation point of the stuck beam, is unknown, solving these nine unknowns is a highly nonlinear problem and the Newton-Rhapson method [30] is applied. To apply the Newton-Rhapson method, there are following four steps: (1) first, guess a set of values for $A_{i}, B_{i}, C_{i}$ and $D_{i}(i=1$ and 2$)$ and $\xi_{1} ;(2)$ for a given concentrated load location of $\xi_{0}$, compare $\xi_{0}$ and $\xi_{1}$ to determine whether the concentrated 


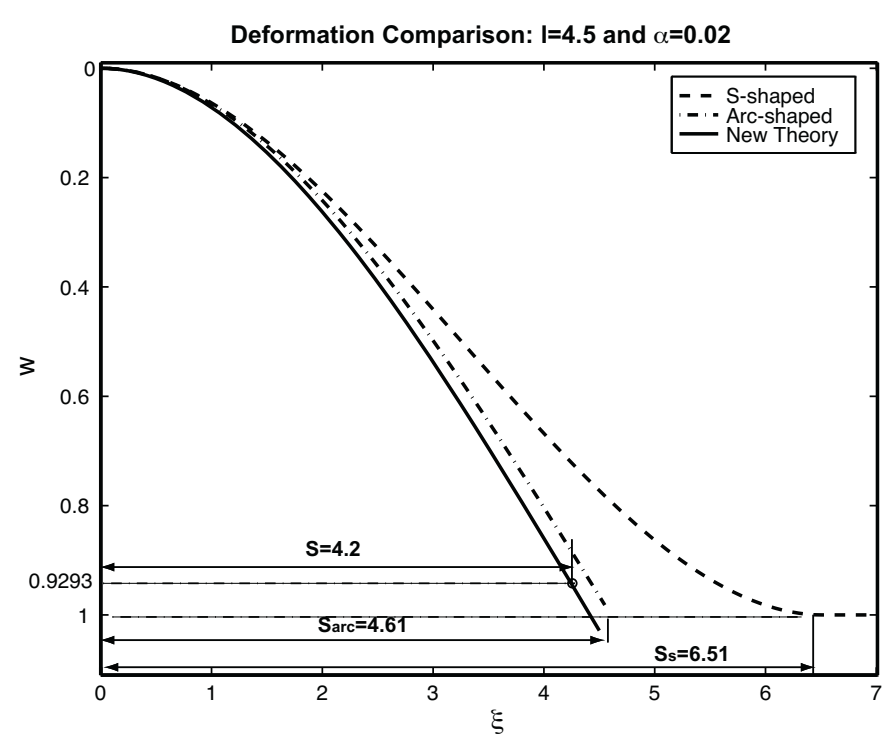

Fig. 3. Deflection comparison of a "chunky" beam ( $l=4.5$ and $\alpha=0.02)$, arc-shape and S-shape.

load is inside the contact zone $\left(\xi_{0}>\xi_{1}\right)$ or outside the contact zone $\left(\xi_{0}>\xi_{1}\right)$ and corresponding solution form (either Eq. (30) or (31)) applies; (3) apply the Newton-Rhapson method to obtain $A_{i}, B_{i}, C_{i}$, $D_{i}$ and $\xi_{1} ;(4)$ go back to step (2) and iterate until the convergence is reached. The results converge very quickly after a few iterations and are not (very) sensitive to the initial guessed values.

\section{Results and discussion}

$h$ is fixed as 1 in this study. The different deflections of a chunky beam with $l=4.5$ and $\alpha=0.02$ under different loadings are shown in Fig. 2. In conjunction with Eqs. (22) and (27), the dimensionless parameter $\alpha$ has the following form: $\alpha=4 B \gamma_{s} /\left(E_{1} I \beta^{2}\right) \propto 24\left(\gamma_{s} / E_{1}\right)(1 / T)$, which in essence indicates the influence of adhesion compared with an elastic one. Here $\gamma_{s} / E_{1}$ is very small, which is around $10^{-9}$ to $10^{-12} \mathrm{~m}$ [31-33] for various materials. Usually the thickness of a MEMS structure is around $10^{-6} \mathrm{~m}$ or larger. $\alpha=0.02$ thus physically indicates a very strong adhesion effect. As indicated by the constraint condition of Eq. (26), all the beam deflections under different loadings separates from the substrate with the same vertical displacement of $h-(\sqrt{\alpha} / 2) \approx$ 0.9293 . However, their contact lengths are different. $\xi_{1}=4.2$ and the contact length is $l-\xi_{1}=0.3$ for $F=Q=0$ (stiction state); $\xi_{1}=3.96$ and the contact length is $l-\xi_{1}=0.54$ for $F=2 \times 10^{-2}, \xi_{0}=l=4.5$ and $Q=0$ (concentrated load inside the contact zone); $\xi_{1}=3.87$ and the contact length is $l-\xi_{1}=0.63$ for $F=2 \times 10^{-2}, \xi_{0}=0.45 l=2.025$ and $Q=0$ (concentrated load outside the contact zone); $\xi_{1}=4.05$ and the contact length is $l-\xi_{1}=0.45$ for $F=0$ and $Q=2 \times 10^{-2}$ (uniformly distributed loading). As seen in Fig. 2, the unstuck or contact length is determined by the load magnitude, the loading type (concentrated or uniformly distributed) and loading location.

The external load does not appear as a parameter in the derivations of arc- and S-shaped deflections as seen in both Appendix A and the previous studies $[7,8,18]$. Again, it is emphasized that stiction here is defined as an attachment state under zero external load. Fig. 3 compares the stiction shape derived by this new method with the arc- and S-shaped deflections, which are described by Eqs. (47) and (48) in Appendix A, respectively. Clearly, our stiction shape ( $F=Q=0$ case) of $l=4.5$ and $\alpha=0.02$ is different from either arcshape or S-shape. The unstuck length predicted by our model is $\xi_{1}=4.2$; the unstuck length of arc-shape is $s_{\text {arc }}=\sqrt[4]{9 h^{2} / \alpha}=4.61$ and that of S-shape is $s_{s}=\sqrt{2} s_{\text {arc }}=6.51$. It is noticed that the

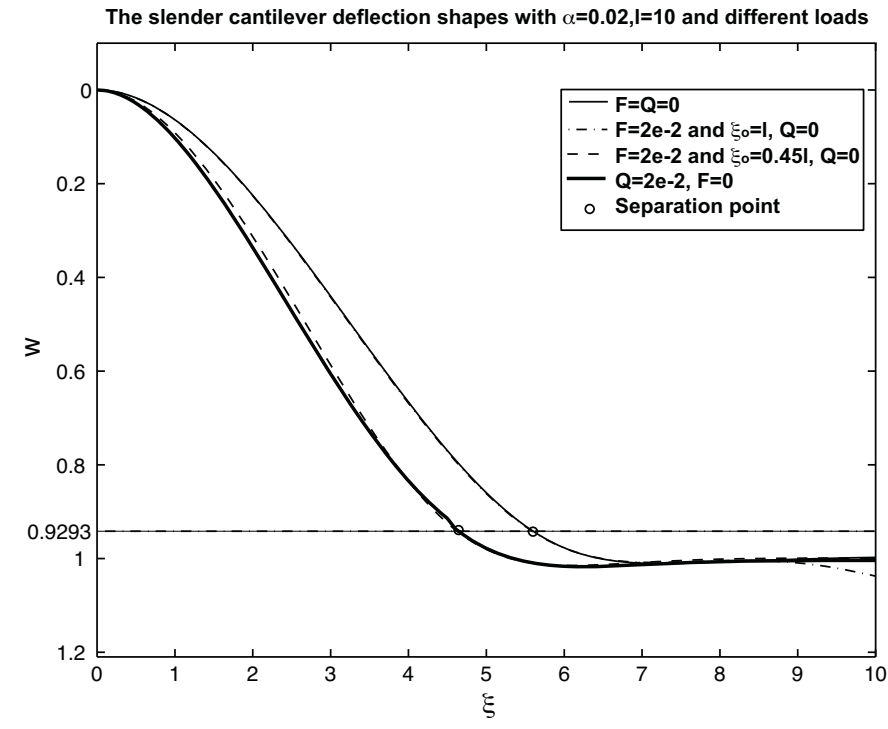

Fig. 4. The deflection shapes of a slender beam with $l=10$ and $\alpha=0.02$ under four different loading scenarios: (1) $F=Q=0$ (stiction state). (2) $F=2 \times 10^{-2}$ and $\xi_{0}=l, Q=0$ (concentrated force inside contact zone). (3) $F=2 \times 10^{-2}$ and $\xi_{0}=0.45 l, Q=0$ (concentrated force outside contact zone). (4) $Q=2 \times 10^{-2}$ and $F=0$ (uniformly distributed load).

beam predicted by our model separates from the substrate with the vertical displacement of $h-(\sqrt{\alpha} / 2) \approx 0.9293$; the vertical displacements at the separation points are $h=1$ for both arc- and $\mathrm{S}$-shapes. The contact zone is now divided into two parts in terms of contact pressure: a zone around the beam free end (i.e., $w(\xi)>$ 1 ) is with compressive pressure and a zone around the contact separation point (i.e., the zone of $0.9293 \leq w(\xi)<1$ ) is with tensile pressure. This resembles the Johnson-Kendall-Roberts (JKR) contact scenario of two spheres: the inner circular zone is with compressive pressure and outer annulus zone is with tensile pressure $[16,17]$. In terms of force equilibrium, the tensile pressure due to adhesion in the zone around the contact separation point balances the restoring forces due to the beam bending and contact deformations. The beam length of $l=4.5$ is smaller than $s_{\text {arc }}$ and $s_{s}$. Therefore, if either an arc-shape or an S-shape assumption is used to predict the beam stiction, the beam with $l=4.5$ and $\alpha=0.02$ cannot adhere to the substrate. In our computation, this $l=4.5$ is the critical length and no stiction can occur with the length shorter than this value. Clearly, in Fig. 3 the arc-shape deflection is a much better approximation than S-shape for the (real) beam stiction shape.

Fig. 4 shows the deflection shapes of a slender beam with $l=10$ and $\alpha=0.02$. Now the deflection curve of $F=Q=0$ (stiction state) overlaps in most part with that of $F=2 \times 10^{-2}, \xi_{0}=l=10$ and $Q=0$ (the concentrated load inside the contact zone); their difference only enlarges around the cantilever free end due to that the concentrated load applied at the end pushes the beam deeper into the substrate; their separation point are around $\xi_{1}=5.49$. The deflection curve of $F=2 \times 10^{-2}, \xi_{0}=0.45 l=4.5$ and $Q=0$ (the concentrated load outside the contact zone) is similar to that of $F=0$ and $Q=2 \times 10^{-2}$ (uniformly distributed loading): there are some differences in the unstuck part and the contact parts are overlapped. For these two loading cases, the separation points are around $\xi_{1}=4.53$. Fig. 5 compares the stiction shape $(F=Q=0)$ of a slender beam with arc- and S-shapes. With the fixed values of $h=1$ and $\alpha=0.02$, the unstuck lengths, $s_{a r c}=4.61$ and $s_{s}=6.51$ of arc-shape and S-shapes, respectively, remain unchanged. Now the S-shape closely matches the deflection curve of the new model. There is only some small difference in the contact area. Again, with the fixed values of $h$ and $\alpha$ the deflection curve of the new model separates from the substrate 


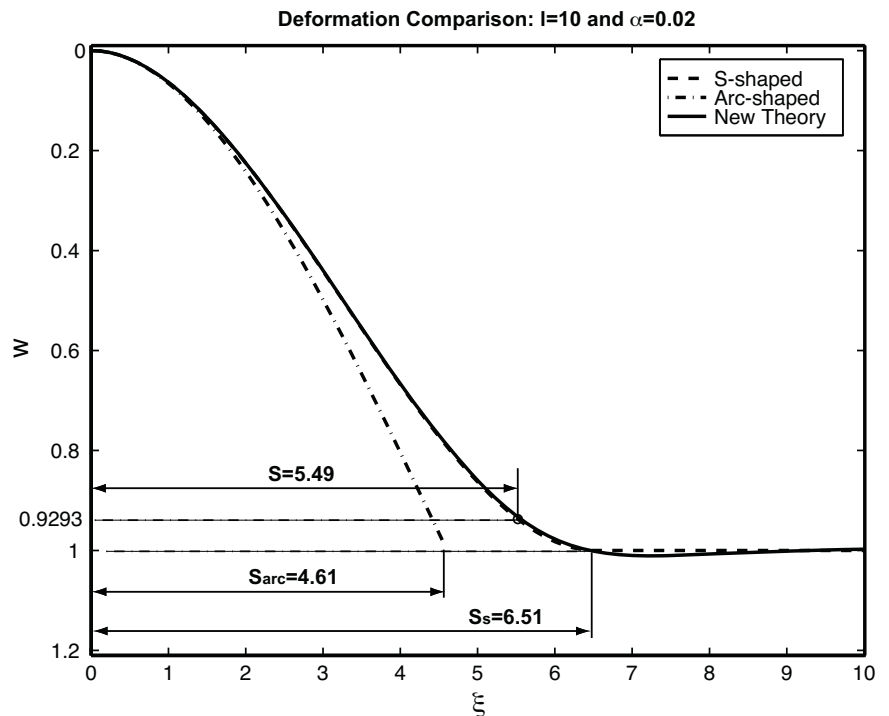

Fig. 5. Deflection comparison of a slender beam $(l=10$ and $\alpha=0.02)$, arc-shape and S-shape.

with the same vertical displacement of 0.9293 . But the unstuck length changes as $\xi_{1}=5.49$. The advantage of the new model is now standing out: rather than prescribing the boundary conditions at the separation point as done by the arc-shape and S-shape, the new model configures its deflection through the matching conditions and constraint conditions. It is also worth emphasizing that though the stiction shape of the new model closely matches the S-shape, their unstuck lengths are different, which has significant impact on the interpretation on the adhesion energy measurement. For example, $s_{S}=6.51$ of S-shape corresponds to $\alpha=0.02$; suppose that $\xi_{1}=5.49$ predicted by our model is the unstuck length measured in experiment and $s_{s}=\xi_{1}=5.49$ corresponds to $\alpha=0.396$ of an S-shape. Therefore, an $\alpha$ error of $98 \%$ results from these two different modelings.

There are three major sources contributing to the deflection difference between the new model and arc/S-shaped deflections: (1) Boundary conditions: at the separation point, the boundary conditions are the hinged ones for arc-shape and clamped ones for S-shape as indicated by Eqs. (37) and (39) in Appendix A, respectively; rather than prescribing the boundary conditions at the separation point, the boundary conditions of the new model are described by the matching condition of Eq. (13) and constraint condition of Eq. (19). (2) The difference in the bending energy: the bending energy of the new model consists of two parts, i.e., the bending energies of both unstuck part and contact/stuck part as given by Eq. (8); the bending energy of both arc- and S-shapes only account the unstuck part as seen in Eqs. (42) and (43) in Appendix A. For arc-shape, the contact is (assumed to be) a point contact and for S-shape, the deflection in the contact area is (assumed to be) flat. The bending energy inside contact area is thus zero with the assumption of arc/S-shaped deflection. However, the deflection is neither exactly arc-shape (as seen in Fig. 2) nor S-shape (as seen in Fig. 5). Therefore, the bending energy inside contact area should not be ignored. (3) The elastic energy due to the contact deformation between the beam and substrate. As seen in Eq. (17), this elastic energy of $U_{F}$ contributes to the total system free energy; however, there is no such elastic energy in the total system free energy of Eq. (45) for both arc-and S-shaped deflections. As mentioned above, arc-shape is an idealized point contact scenario. As seen in Fig. 2 the beam part near the cantilever free end penetrates into the substrate (i.e., $w>h$ ) and this contact energy is not a small amount as

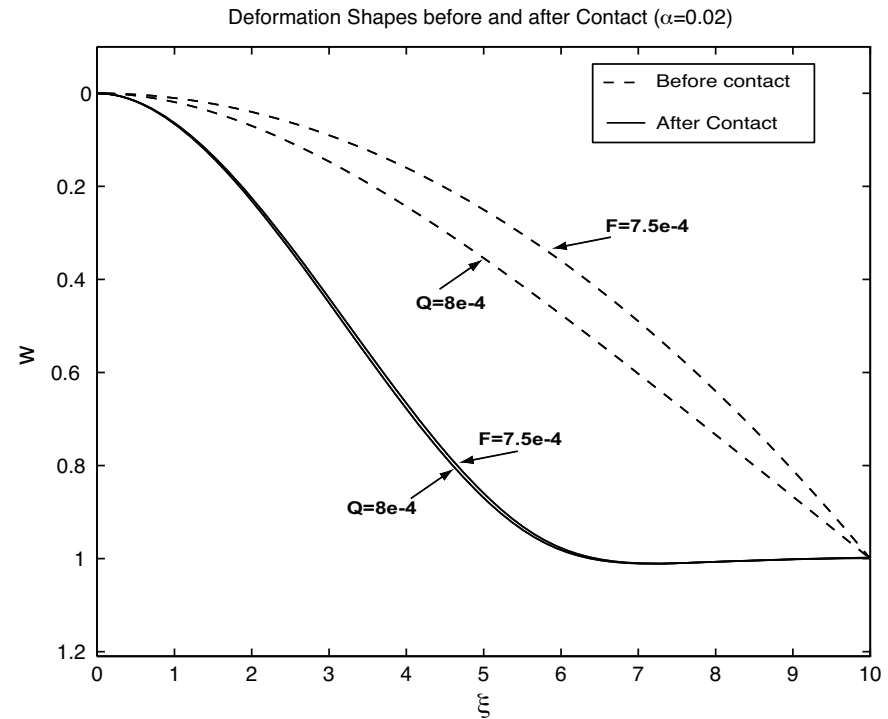

Fig. 6. The deflection shapes of a slender beam $(l=10$ and $\alpha=0.02)$ before and after contact for two loading scenarios: $F=7.5 \times 10^{-4}, Q=0$ (concentrated load only) and $F=0, Q=8 \times 10^{-4}$ (distributed load only).

compared with the bending energy of the unstuck part. Therefore, this contact elastic energy should not be ignored, either.

Fig. 6 compares the beam deflection shapes just before and after contact. The beam is with $l=10$ and $\alpha=0.02$. Before contact, the beam is governed by Eq. (4). If we set $w(l)=h, \xi=l$ and $\xi_{0}=l, F$ is found from Eq. (23) for the free-standing beam as follows:

$F=\frac{3 h}{4 l^{3}}=7.5 \times 10^{-4}$

This $F$ value physically means if only a concentrated load with this magnitude is applied at the cantilever free end, the vertical displacement of the free end is $h$, which just touches the substrate. Similarly, if only a uniformly distributed load is applied and the beam free end just touches the substrate, $Q$ is found as follows:

$Q=\frac{8 h}{l^{4}}=8 \times 10^{-4}$

After the contact is initiated, Eq. (28) or (29) becomes the governing equation depending on whether the concentrated load is inside or outside the contact area. As seen in Fig. 6 there are abrupt deflection shape changes in both the concentrated loading and uniformly distributed loading cases. It is also noticed that once the contact starts, the contact length is a finite one rather than a point contact, which has been observed by Liu [34]. When a microcantilever is rinsed by a liquid or under an electrostatic loading, the (attractive) nonlinear capillary/electrostatic force is the driving force to push down the cantilever to bring it into contact with the substrate. The competition between the capillary/electrostatic force and beam elastic restoring force results in an instability called pull-in instability [35], which leads to an abrupt contact of microstructure with substrate [6,35]. Mathematically, the abrupt change of deflection shape is due to the governing equation change from the free-standing state to an adhesive contact state. Physically, in the free-standing state, there are no adhesion and contact deformation between the beam and substrate.

The new model is also capable of studying the de-adherence of microcantilever by simply changing the $F$ or $Q$. Here the positive $F$ and $Q$ are the compressive (pushing down) force and negative ones are the tensile (pulling up) force. A uniformly distributed load is applied and the beam length is $l=10$ in Fig. 7. Fig. 7(a) plots the cantilever free end displacement of $w(l)$ as a function of $Q$ for different $\alpha$ s. Interestingly, the cantilever free end displacements decrease 
(a) Cantilever Tip Displacement versus Q

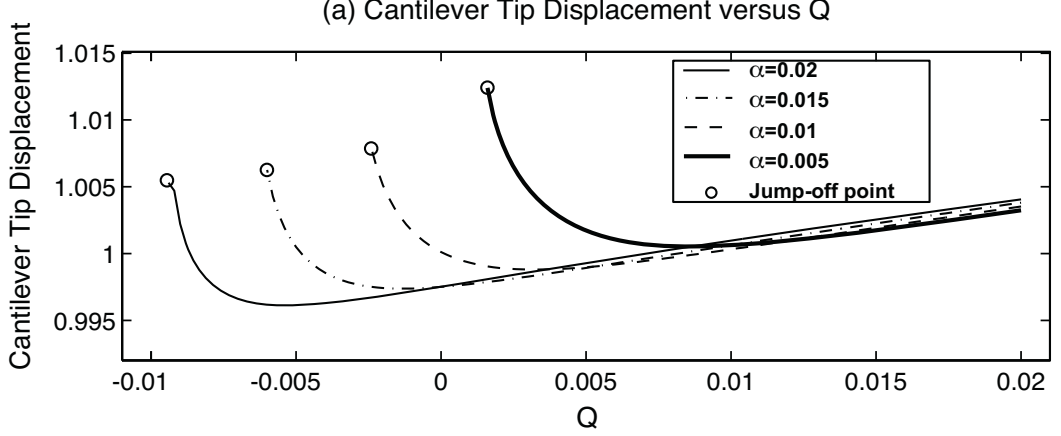

(b) Contact Length versus $Q$

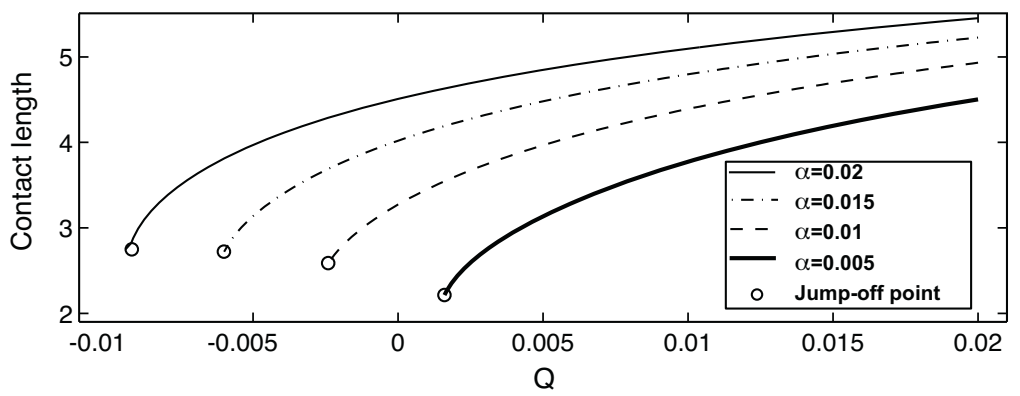

Fig. 7. Jump-off of a stuck slender beam $(l=10)$ : (a) The cantilever tip displacement as a function of $Q$. (b) The contact length as a function of $Q$.

and then increase with the decrease of $Q$. With the change of $Q$, the beam changes its equilibrium configuration: when $Q$ is compressive and $\alpha$ is relatively large, the beam deforms like an S-shape as shown in Fig. 5; when the beam is about to separate/jump-off from the substrate ( $Q$ is tensile or even compressive for small $\alpha$ cases), the beam deforms like an arc-shape as seen in Fig. 3 and the beam part around the free end penetrates deeper into the substrate, which is reason for the increase of the cantilever free end displacement with the decrease of $Q$ in the stage about to separate. Similarly, Jones et al. [14] found from their load-deflection experimental data that the stiffness of a stuck cantilever changes in both loading and unloading stages, which indicates the transition between an arc-shape and an S-shape. In contrast, the contact length of $l-\xi_{1}$ decreases monotonously with the decrease of $Q$ as shown in Fig. 7(b). It is noticed that around separation the slopes of both Fig. 7(a) and (b) become very steep, which physically leads to an abrupt jump-off behavior. In the adhesive contact of spheres, the JKR theory also predicts such abrupt jump-off behavior during the separation process $[16,17]$. The beam jumps-off at $Q=1.6 \times 10^{-3}$ for $\alpha=5 \times 10^{-3}$, which physically means that there is no stiction state for this small $\alpha$ case and an extra compressive external load is needed to keep the beam in contact with the substrate; the beam jumps-off at $Q=-2.4 \times 10^{-3}$ for $\alpha=1 \times 10^{-2}$, at $Q=-6 \times 10^{-3}$ for $\alpha=1.5 \times 10^{-2}$ and at $Q=-9.4 \times 10^{-3}$ for $\alpha=2 \times 10^{-2}$. Clearly, except the case of $\alpha=5 \times 10^{-3}$, a larger tensile force is required to separate the stuck beam with a larger $\alpha$ from the substrate. It is not hard to understand this from a viewpoint of fracture mechanics $[8-10,14,20]$ on the deadherence of microcantilever: the separation of a stuck cantilever from a substrate in essence is to make one interface into two surfaces, which increases surface energy and more external work/force is thus required for a larger $\alpha\left(\gamma_{s}\right)$. Similarly, the JKR contact theory also predicts that the tensile peel-off force is proportional to $\gamma_{s}$ [16,17].

Only the uniformly distributed loading is selected in Fig. 7 to study the beam de-adherence because the beam deflection under a uniformly distributed loading can always keep the noncontact-contact configuration as prescribed by Eq. (7). When a tensile concentrated load is applied, the noncontact-contact configuration of deflection can break. As seen in Fig. 8, a concentrated force is applied at the free end of a cantilever with $l=10$ and $\alpha=0.02$. When $F$ is compressive or tensile with $F>-3.4 \times 10^{-2}$, this noncontact-contact configuration still remains. Once a larger tensile force (i.e., $F<-3.4 \times 10^{-2}$ ) is applied, the cantilever free end will separate from the substrate: a noncontact-contact-noncontact configuration is formed, which makes the governing equation of Eq. (28) or Eq. (29) invalid. The governing equation for the noncontact-contact-noncontact configuration as seen in Refs. $[24-26,29]$ needs to be re-derived for the adhesive contact of a cantilever beam.

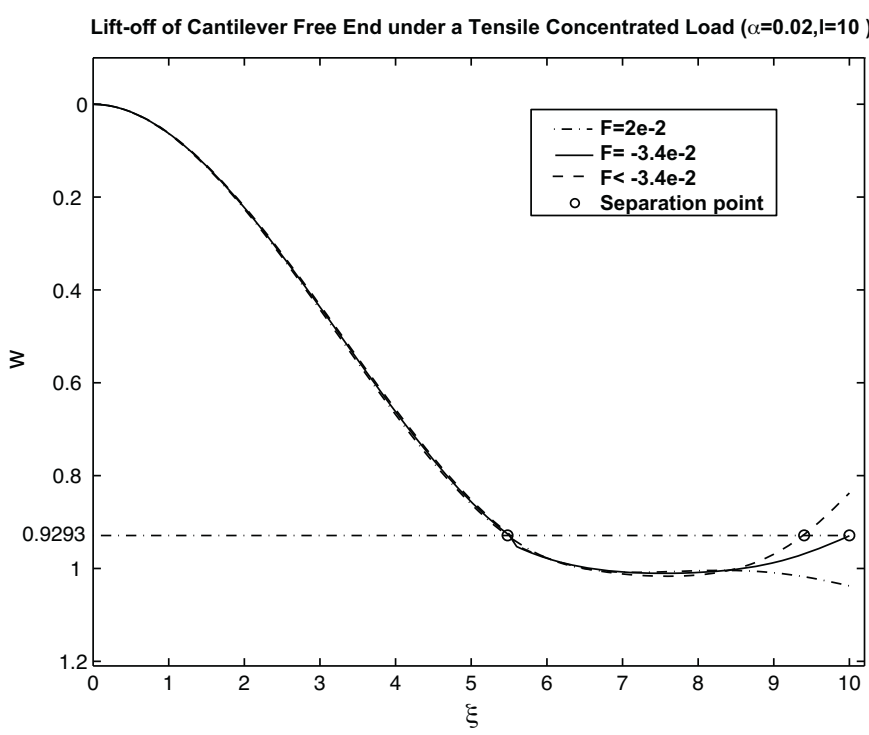

Fig. 8. Separation of the cantilever free end from the substrate and a deflection shape with a noncontact-contact-noncontact configuration under a tensile concentrated load. 


\section{Summary}

A general approach of studying the microcantilever stiction is presented. The principle of virtual work is used to derive the governing equation by assuming the deflection shape with a noncontact-contact configuration. The minimization of the system free energy results in the constraint condition, which determines the separation point. There are two sets of the governing equation depending on whether the concentrated load is inside or outside the contact zone. The governing equations becomes invalid when a noncontact-contact-noncontact zone is formed by a tensile concentrated load. This new approach shows that the deflection shape of a stuck cantilever beam is a function of the beam dimensions and mechanical properties, gap distance, adhesion, loading type and magnitude. The transition and change of the cantilever deflection shape are demonstrated by changing the beam dimensions and loadings. In comparison, the arc-shaped and S-shaped deflections only offer an approximation for the zero loading case, which deviates more or less from the one predicted by this approach. The difference of the deflection shapes has a direct impact on the calculation of the system energy and thus the interpretation of experimental data. In essence, this new approach offers a more accurate model on the stuck cantilever by not prescribing its deflection shape.

\section{Acknowledgments}

This work is supported by the National Natural Science Foundation of China (NSFC, Grant Nos. 10721202 and 10772180), Ministry of Science and Technology (MOST Grant No. 2010CB631004) and National Basic Research Program of China (973 Program, Grant No. 2007CB310500).

\section{Appendix A. Stiction with an arc-shape and an S-shape}

Fig. 1(b) shows the arc-shaped and S-shaped deformations. Here stiction is defined as a state with no external load, the following governing equation holds:

$E_{1} I \frac{d^{4} W}{d x^{4}}=0$

Eq. (35) is readily solved as follows:

$W(x)=A x^{3}+B x^{2}+C x+D$

$A, B, C$ and $D$ are the four unknown constants to be determined by the boundary conditions.

For an arc-shaped beam, one end is fixed and the other is hinged. Therefore, the following boundary conditions hold:

$W(0)=0, \quad \frac{d W}{d x}(0)=0, \quad W(S)=H, \quad \frac{d^{2} W}{d x^{2}}(S)=0$

$S$ is the unstuck length. $W(x)$ of an arc-shaped beam is then solved as follows [9]

$W(x)=-\frac{H}{2 S^{3}} x^{3}+\frac{3 H}{2 S^{2}} x^{2}$

For an S-shaped beam, both ends are fixed. Therefore, the following boundary conditions hold:

$W(0)=0, \quad \frac{d W}{d x}(0)=0, \quad W(S)=H, \quad \frac{d W}{d x}(S)=0$

$W(x)$ of an S-shaped beam is then solved as follows [9]

$W(x)=-\frac{2 H}{S^{3}} x^{3}+\frac{3 H}{S^{2}} x^{2}$
In both Eqs. (38) and (40), $S$ is unknown. To solve $S$, the system free energy is needed. The beam bending energy, $U_{b}$ is given as follows:

$U_{b}=\frac{E_{1} I}{2} \int_{0}^{S}\left(\frac{d^{2} W}{d x^{2}}\right)^{2} d x$

For arc-shape, substitute Eq. (38) into Eq. (42) and we have

$U_{b}^{a r c}=\frac{E_{1} I}{2} \int_{0}^{S}\left(-\frac{3 H}{S^{3}} x+\frac{3 H}{S^{2}}\right)^{2} d x=\frac{3 E_{1} I}{2} \frac{H^{2}}{S^{3}}=\frac{E_{1} B T^{3} H^{2}}{4 S^{3}}$

Here $I=B T^{3} / 6$ ( $2 B$ is the beam width). For S-shape, substitute Eq. (40) into Eq. (42) and we have

$U_{b}^{S}=\frac{E_{1} I}{2} \int_{0}^{S}\left(-\frac{12 H}{S^{3}} x+\frac{6 H}{S^{2}}\right)^{2} d x=\frac{6 E_{1} I H^{2}}{S^{3}}=\frac{E_{1} B T^{3} H^{2}}{S^{3}}$

The surface energy $U_{S}$ is as follows:

$U_{S}=-2 B \gamma_{S}(L-S)$

Again, $2 B$ here is the beam width and $L-S$ is the beam contact length. The system total free energy is

$U_{t}=U_{b}+U_{S}= \begin{cases}\frac{E_{1} B T^{3} H^{2}}{4 S^{3}}-2 B \gamma_{s}(L-S), & \text { arc-shape } \\ \frac{E_{1} B T^{3} H^{2}}{S^{3}}-2 B \gamma_{S}(L-S), & \text { S-shape }\end{cases}$

Compared with the total energy of Eq. (17), besides the difference in the bending energy, $U_{t}$ here does not have $U_{F}$, the elastic energy due to the contact between the beam and substrate. For equilibrium, $d U_{t} / d S=0$, which yields $S$ for arc-shape and S-shape as follows [5]

$\begin{cases}S^{4}=S_{\text {arc }}^{4}=\frac{3}{8} \frac{E_{1} H^{2} T^{3}}{\gamma_{S}}=\frac{9 E_{1} I H^{2}}{4 B \gamma_{s}}, & \text { arc-shape } \\ S^{4}=S_{S}^{4}=\frac{3}{2} \frac{E_{1} H^{2} T^{3}}{\gamma_{S}}=\frac{9 E_{1} I H^{2}}{B \gamma_{s}}, & \text { S-shape }\end{cases}$

Clearly, $S_{S}=\sqrt{2} S_{\text {arc }}=\sqrt[4]{\frac{3}{2} \frac{E_{1} H^{2} T^{3}}{\gamma_{s}}}$ [5]. It is also noticed from the above equation that the unstuck lengths of both $S_{\text {arc }}$ and $S_{s}$ are independent of the beam length $L$. For S-shape, the unstuck part is described by Eq. (40) and stuck part $\left(S_{S} \leq X \leq L\right)$ is a flat one as shown in Fig. 1(b). However, there is a problem for the arc-shaped deformation if $L>S_{\text {arc }}$. The rotation angle of arc-shape at the hinged end is not zero, the beam part of $S_{\text {arc }} \leq X \leq L$ has to penetrate into the substrate, which causes large contact energy and is not accounted by Eq. (45). When $L \approx S_{\text {arc }}$, such penetration is allowed as shown in Fig. 3; When $L$ is much larger than $S_{\text {arc }}$, the arc-shaped deflection is not allowed. Arc-shape in essence is an idealized deflection shape of point contact with $L=S_{\text {arc }}$.

The arc-shaped deflection of Eq. (38) is now nondimensionalized as follows:

$w(\xi)=-\frac{h}{2 s_{\text {arc }}^{3}} \xi^{3}+\frac{3 h}{2 s_{\text {arc }}^{2}} \xi^{2}$

Here $s_{\text {arc }}=\beta S_{\text {arc }}=\sqrt[4]{9 h^{2} / \alpha}$. The S-shaped deflection of Eq (40) is nondimensionalized as follows:

$w(\xi)=-\frac{2 h}{s_{s}^{3}} \xi^{3}+\frac{3 h}{s_{s}^{2}} \xi^{2}$

Here $s_{s}=\beta S_{s}=\sqrt[4]{36 h^{2} / \alpha}=\sqrt{2} s_{\text {arc }}$

\section{References}

[1] W.M. van Spengen, R. Puers, I. De Wolf, A physical model to predict stiction in MEMS, J. Micromech. Microeng. 12 (2002) 702-713.

[2] W.M. van Spengen, R. Puers, I. De Wolf, The prediction of stiction failures in MEMS, IEEE Trans. Dev. Mater. Reliab. 3 (2003) 167-172. 
[3] R. Maboudian, R.T. Howe, Critical review: adhesion in surface micromechanical structures, J. Vac. Sci. Technol. B 15 (1997) 1-20.

[4] N. Tas, T. Sonnernberg, H. Jansen, R. Legtenberg, M. Elwenspoek, Stiction in surface micromaching, J. Micromech. Microeng. 6 (1996) 385-397.

[5] F. Yang, Contact deformation of a micromechanical structure, J. Micromech. Microeng. 14 (2002) 263-268.

[6] C.H. Mastrangelo, C.H. Hsu, Mechanical stability and adhesion of microstructures under capillary forces - part I: basic theory, J. Microelectromech. Syst. 2 (1993) 33-43.

[7] R. Legtenberg, T. Tilmans, J. Elders, M. Elwenspoek, Stiction of surface micromachined structures after rinsing and drying: model and investigation of adhesion mechanisms, Sens. Actuators A 43 (1994) 230-238.

[8] M.P. de Boer, T.A. Michalske, Accurate method for determining adhesion of cantilever beams, J. Appl. Phys. 86 (1999) 817-827.

[9] M.P. de Boer, J.A. Knapp, T.M. Mayer, T.A. Michalske, The role of interfacial properties on MEMS performance and reliability, Proc. SPIE 3825 (1999) 2-15.

[10] J.A. Knapp, M.P. de Boer, Mechanics of microcantilever beams subjected to combined electrostatic and adhesive forces, J. Microelectromech. Syst. 11 (2002) 754-764.

[11] Y. Zhang, Y.P. Zhao, Static study of cantilever beam stiction and electrostatic force influence, Acta Mech. Solida Sin. 17 (2004) 104-112.

[12] Y. Zhang, Y.P. Zhao, Vibration of an adhered microbeam under a periodically shaking electrical force, J. Adhes. Sci. Technol. 19 (2005) 799-815.

[13] A. Savkar, K.D. Murphy, Z.C. Leseman, T.J. Mackin, M.R. Begley, On the use of structural vibrations to release stiction failed MEMS, J. Microelectromech. Syst. 16 (2007) 163-173.

[14] E.E. Jones, M.R. Begley, K.D. Murphy, Adhesion of micro-cantilever subjected to mechanical point loading: modeling and experiments, J. Mech. Phys. Solids 51 (2003) 1601-1622.

[15] H.H. Yu, Z. Suo, A model of wafer bonding by elastic accommodation, J. Mech. Phys. Solids 46 (1998) 829-844.

[16] K.L. Johnson, Contact Mechanics, Cambridge University Press, Cambridge, 1985 (Chapter 5).

[17] Y. Zhang, Transitions between different contact models, J. Adhes. Sci. Technol. 22 (2008) 699-715

[18] C.H. Mastrangelo, C.H. Hsu, Mechanical stability and adhesion of microstructures under capillary forces - part II: experiments, J. Microelectromech. Syst. 2 (1993) 44-55.

[19] Y.P. Zhao, L.S. Wang, T.X. Yu, Mechanics of adhesion in MEMS - a review, J. Adhes. Sci. Technol. 17 (2003) 519-546.
[20] J.W. Rogers, T.J. Mackin, L.M. Phinney, A thermomechanical model for adhesion reduction of MEMS cantilevers, J. Microelectromech. Syst. 11 (2002) 512-520.

[21] L. Meirovitch, Analytical Methods in Vibrations, Macmillan Publishing Co. Inc. New York, 1967 (Chapter 6).

[22] J.W. Obreimoff, The splitting strength of mica, Proc. R. Soc. London Ser. A 127 (1930) 290-297.

[23] A. Hariri A, J.W. Zu, R. Ben Mrad, Modeling of dry stiction in microelectro-mechanical systems (MEMS), J. Micromech. Microeng. 16 (2006) 1195-1206.

[24] Y. Weitsman, On foundations that reacts in compression only, J. Appl. Mech. 37 (1970) 1019-1030.

[25] Y. Zhang, K.D. Murphy, Response of a finite beam in contact with a tensionless foundation under symmetric and asymmetric loading, Int. J. Solids Struct. 41 (2004) 6745-6758.

[26] Y. Zhang, Tensionless contact of a finite beam resting on Reissner foundation Int. J. Mech. Sci. 50 (2008) 1035-1041.

[27] M.A. Biot, Bending of an infinite beam on an elastic foundation, J. Appl. Mech. 4 (1937) 1-7.

[28] A. Ghatak, L. Mahadevan, M.K. Chaudhury, Measuring the work of adhesion between a soft confined film and a flexible plate, Langmuir 21 (2005) 1277-1281.

[29] Y. Zhang, Extracting nanobelt mechanical properties from nanoindentation, J. Appl. Phys. 107 (2010) 123518

[30] W.H. Press, S.A. Teukolsky, W.T. Vetterling, B.P. Flannery, Numerical Recipes in Fortran, 2nd ed., Cambridge University Press, Cambridge, 1992 (Chapter 9).

[31] R.E. Miller, V.B. Shenoy, Size-dependent elastic properties of nanosized structural elements, Nanotechnology 11 (2000) 139-147.

[32] M. Giri, D.B. Bousfield, W.N. Unertl, Dynamic contacts on viscoelastic film: work of adhesion, Langmuir 17 (2001) 2973-2981.

[33] Y. Sun, B. Akhremitchev, G.C. Walker, Using the adhesive interaction between atomic force microscopy tips and polymer surfaces to measure the elastic modulus of compliant sample, Langmuir 20 (2004) 5837-5845.

[34] J.L. Liu, Theoretical analysis on capillary adhesion of microsized plates with a substrate, Acta Mech. Sin. 26 (2010) 217-223.

[35] Y. Zhang, Y.P. Zhao, Numerical and analytical study on the pull-in instability of micro-structure under electrostatic loading, Sens. Actuators A 127 (2006) $366-380$. 